\title{
Correlations in $e^{+} e^{-}$interactions
}

\author{
Tariq Aziz ${ }^{\dagger}$ \\ EP-L3, CERN, 1211 Geneva 23, Switzerland \\ E-mail: tariq.aziz@cern.ch,
}

\begin{abstract}
Experimental studies on two-particle and multi-particle correlation in $e^{+} e^{-}$ interactions performed by the L3, OPAL and SLD collaborations are summarised. Interesting results on Bose-Einstein correlation, Fermi-Dirac correlation, Factorial Cumulants and Rapidity Gap correlation are discussed.
\end{abstract}

\section{Introduction}

Correlation studies in multi-particle production in a variety of interactions have been studied over many years [i1].1. These studies have played a vital role in understanding the production dynamics in these interactions. The present renewal of interest, however, is more due to the fact that understanding correlations in these processes is crucial for understanding precision measurements of $\mathrm{W}$ boson mass at $e^{+} e^{-}$colliders where a pair of W's produced can lead to four jets of hadrons. The fragmenting partons can interfere and this interference can manifest via change in correlations. Variety of correlation studies have been reported at this conference. We restrict to: Bose-Einstein correlations [2],

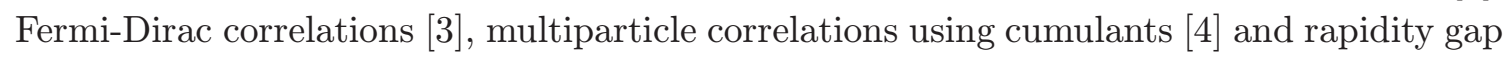
correlations 院. As a set they cover both short range and long range correlations.

\section{Bose-Einstein and Fermi-Dirac Correlations}

Bose-Einstein and Fermi-Dirac correlations study the effect of two-particle wave functions (pair of identical bosons or fermions) when these particles are in close proximity. As a result of the interference either the density of pairs increases (BEC) or decreases (FDC). The range of this effect provides information about the space-time structure of the emitting source. ${ }^{1}$ The effect is analysed using two particle correlation function expressed in terms

\footnotetext{
${ }^{*}$ Speaker.

${ }^{\dagger}$ On behalf of the L3, OPAL and SLD Collaborations

${ }^{1} \mathrm{~A}$ precise understanding of the effect is far from satisfactory, although experimental observations have accumulated over four decades "6.6".
} 
of four-momenta of the two particles $p_{1}, p_{2}$ as:

$$
C\left(p_{1}, p_{2}\right)=\frac{\rho\left(p_{1}, p_{2}\right)}{\rho_{0}\left(p_{1}, p_{2}\right)}
$$

where $\rho_{0}\left(p_{1}, p_{2}\right)$, the two particle density in the absence of BE/FD correlation obtained using $\mathrm{MC}$ sample. This acts as normalisation scale instead of using single particle density functions $\rho_{1}\left(p_{1}\right) \cdot \rho_{1}\left(p_{2}\right)$ from data and takes into account the residual correlations, if any. The phase space proximity is measured using 4-momentum difference/gap:

$$
Q^{2}=-\left(p_{1}-p_{2}\right)^{2}=M_{12}^{2}-4 m^{2}
$$

where $M_{12}$ is the invariant mass of the pair and $m$ is the mass of the individual particle. Assuming spherical particle emission source with a Gaussian density the two particle correlation is parametrised as:

$$
C\left(p_{1}, p_{2}\right)=C(Q)=\mathcal{N}\left(1+\xi \lambda e^{-Q^{2} R^{2}}\right)
$$

where $R$ is radius of the source, $\lambda$ the strength of correlation/chaoticity and $\xi$ is +1 for bosons and -1 for fermions. Here $\mathcal{N}$ is the normalisation coefficient.

The L3 collaboration has studied Bose-Einstein correlation in $\pi^{0} \pi^{0}$ from $\mathrm{Z}$ decays for the first time, and compared with charged pion correlation. The excellent BGO crystal

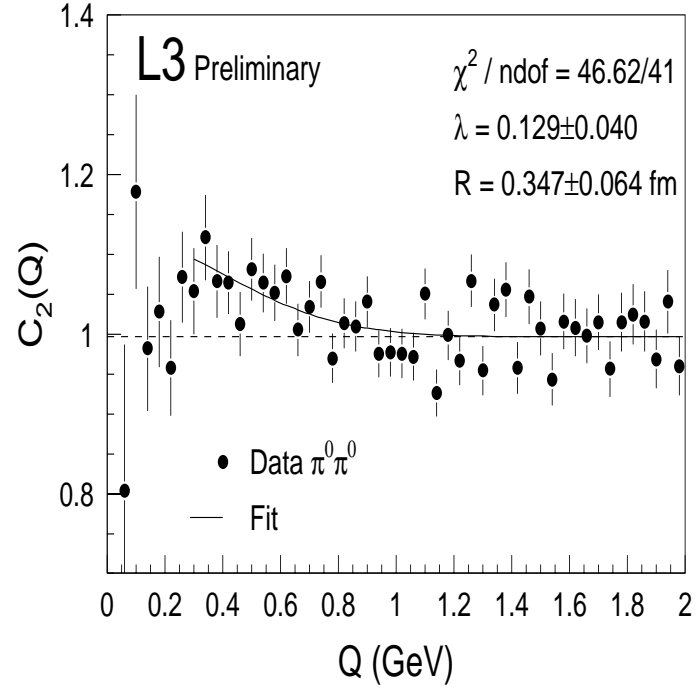

Figure 1: $C_{2}(Q)$ distribution for $\pi^{0} \pi^{0}$. Fit is restricted to $Q>300 \mathrm{MeV}$ as efficiencies at lower values are less reliable.

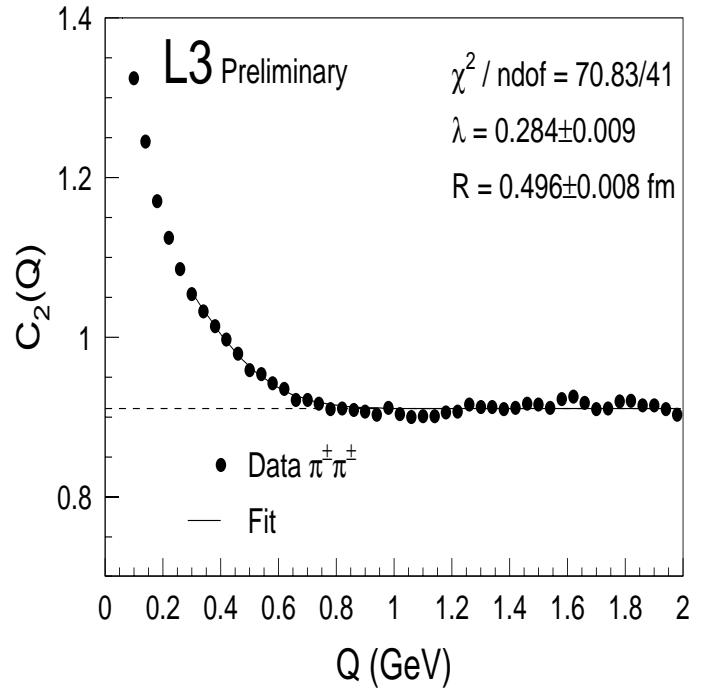

Figure 2: $C_{2}(Q)$ distribution for charged pion pair. For ease of comparison the fit range is kept the same as for $\pi^{0} \pi^{0}$.

electromagnetic calorimeter in L3 has been used for reconstructing $\pi^{0}$ where $\sigma\left(M_{\gamma \gamma}\right) \simeq$ $7 \mathrm{MeV}$. The pair $\pi^{0} \pi^{0}$ is formed in such a way that no photon cluster is common to both $\pi^{0}$ 's at the same time, in order to determine the the four momentum difference $Q$. The $\pi^{0} \pi^{0}$ component of the $Q$ distribution is estimated by two-dimensional mass spectrum for every bin in $Q$ [i] and this leads to $\pi^{0} \pi^{0}$ probability to extract the BE correlation. The result of such pairing and the fit to the data to extract correlation parameters in equation (2.3) is shown in figure ${ }_{-1}^{1}$ In figure $\underset{2}{\overline{2}}$ result of similar study for charged pions is shown. 
A fit to equation $(\overline{2} \cdot \overline{3})$, for $\pi^{0} \pi^{0}$ correlation data yields $\lambda_{\pi^{0} \pi^{0}}=0.129 \pm 0.040_{-0.040}^{+0.038}$ and $R_{\pi^{0} \pi^{0}}=0.347 \pm 0.064_{-0.057}^{+0.075} \mathrm{fm}$. Whereas a fit for charged pion correlation data yields, $\lambda_{\pi^{ \pm} \pi^{ \pm}}=0.284 \pm 0.009_{-0.050}^{+0.019}$ and $R_{\pi^{ \pm} \pi^{ \pm}}=$ $0.496 \pm 0.008_{-0.018}^{+0.044} \mathrm{fm}$. These results indicate that $\pi^{0} \pi^{0}$ source has smaller dimension than the charged pion, $\pi^{ \pm} \pi^{ \pm}$, source. This result is in qualitative agreement with the string fragmentation model [i 81 ], as two neutral pions can stay at adjacent hadronisation vertices, but two same charge pions can not.

The OPAL collaboration has studied FermiDirac correlations using anti-protons [3i] $]$. Using $d E / d X$ measurements they select $6500 \bar{p} \bar{p}$ pairs with a purity of $\simeq 70 \%$ from hadronic $\mathrm{Z}$ decays.

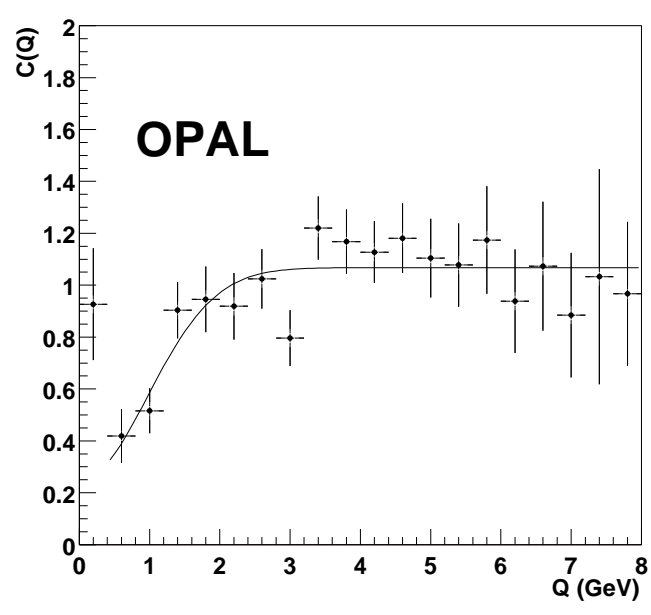

Figure 3: Correlation function $C(Q)$ for $\bar{p} \bar{p}$. The line shows the result of the fit to equation $(2.3 i)$.

The resulting correlation as a function of four momentum difference of the pair is shown in figure $\overline{3}_{\mathbf{r}}^{i}$ Fitting equation $(\overline{2} . \overline{3}$ i) for $\xi=-1$ leads to $\lambda \bar{p} \bar{p}=0.75 \pm 0.15($ stat $)+0.25($ syst $)$ and $R_{\bar{p} \bar{p}}=0.141 \pm 0.033$ (stat) \pm 0.036 (syst) fm. This is an excellent measurement and confirms the mass hierarchy for the emission source $R_{\pi \pi}>R_{K K}>R_{p p}>R_{\Lambda \Lambda}$, when other measurements are considered together.

\section{Study of Factorial Cumulants}

In another interesting study of correlations, OPAL collaboration has considered factorial cumulants [igi $]$ as a measure of genuine correlations ${ }^{2}$ for 2-, 3- and 4-particles. The study is performed by partitioning the phase space into cells of rapidity, $y$, azimuth angle, $\Phi$, and transverse momentum, $P_{T}$. The smallest cells correspond to $\delta y=0.01, \delta \Phi=0.9^{\circ}$ and $\delta\left(\ell n P_{T}\right) \simeq 0.008$. One, two and three dimensional cells have been considered for the multiparticle correlation studies. As an example two, three and four particles correlations in terms of comulants $K_{2}, K_{3}$ and $K_{4}$ in cells of $y \times \Phi$ and $y \times \Phi \times P_{T}$ are shown in figure data is compared with simulation using Pythia generator and introducing Bose-Einstein correlation. The data strongly favour the need to include BEC. What is even more interesting is that introducing only two-particle BEC explains even the higher multiplets, indicating that higher multiplet correlations are essentially driven by two-particle correlations. This is more explicitly seen in figure are plotted as function of $K_{2}$. The data satisfy an elegant relationship $\ell n K_{q}=a_{q}+r_{q} \ell n K_{2}$ and show that the slope $r_{q}$ increases with the order of the cumulant. More interestingly this also confirms that the multiparticle-correlations are driven by two-particle correlations, as noticed earlier. Expectations from the Negative Binomial multiplicity distribution are not favoured by the data.

\footnotetext{
${ }^{2}$ By construction whenever a particle within a multiplet is statistically independent of the others, the cumulant vanishes.
} 


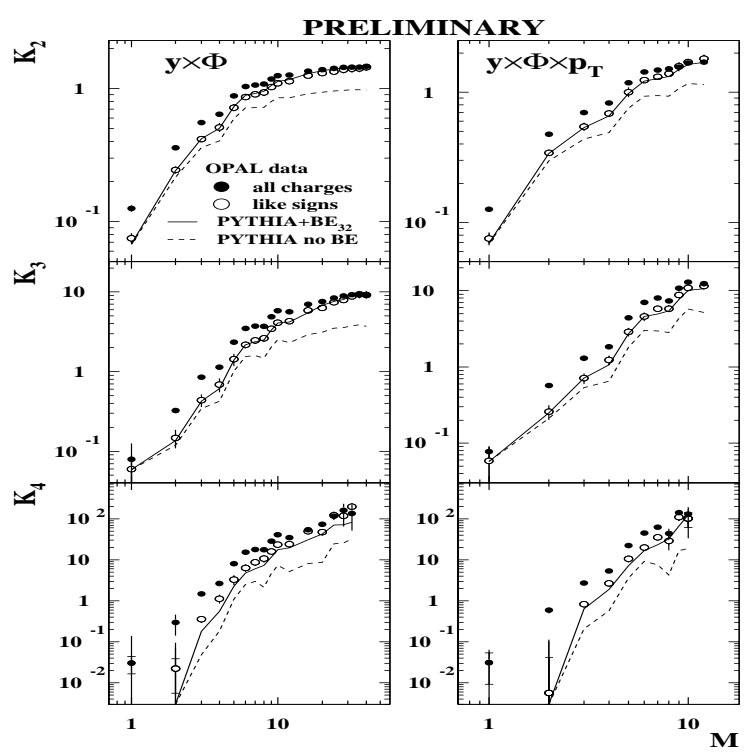

Figure 4: OPAL study of cumulants $K_{q}$ in two and three dimensional cells for all-sign and like-sign multiplets. The simulation is compared only for like-sign multiplets.

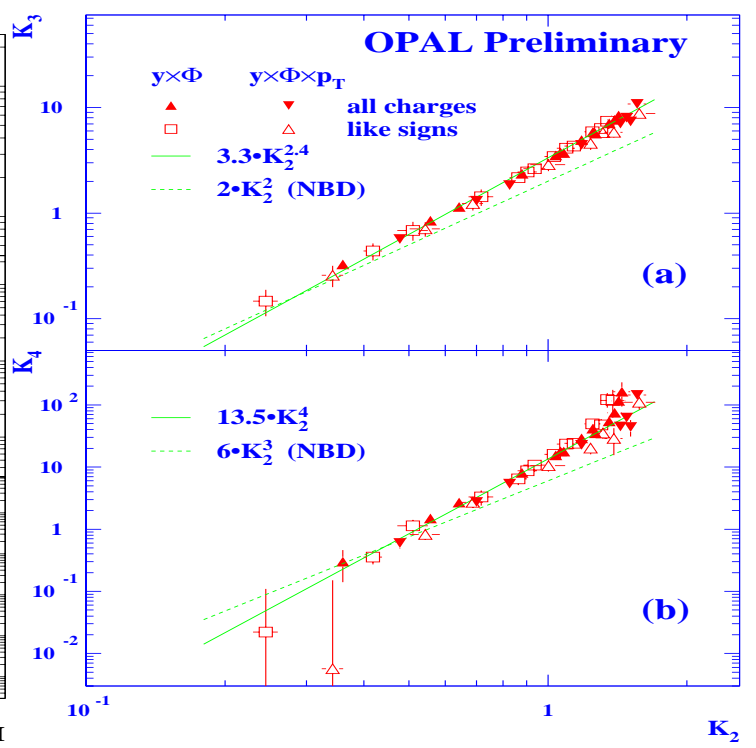

Figure 5: Dependence of 3- and 4-particle cumulants on 2-particle cumulants for all charge multiplets and same charge multiplets in cells of $y \times \Phi$ and $y \times \Phi \times P_{T}$.

\section{Rapidity Gap Correlations}

The SLD collaboration has studied two particle rapidity gap correlations, where Chrenkov Ring Imaging Detector (CRID) has been extensively used to identify $\pi / K / p$. Taking event thrust as the reference axis the particle rapidity $Y=\frac{1}{2} \ell n \frac{\left(E+p_{\|}\right)}{\left(E-p_{\|}\right)}$has been used to study two particle rapidity gap, $|\Delta Y|=\left|Y_{1}-Y_{2}\right|$, correlation for any two particles of same flavour, different flavour, same charge and opposite charge. Studies have also been made in events tagged as heavy flavour events like $Z \rightarrow c \bar{c}$ and $Z \rightarrow b \bar{b}$. As example we show

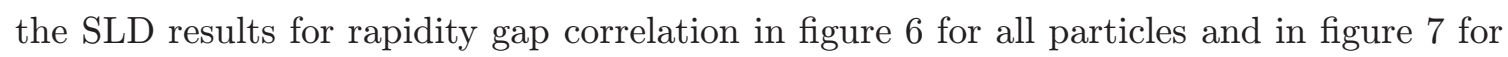
hard particles of momentum in excess of $9 \mathrm{GeV} /$ c. Rapidity gaps $|\Delta Y|=\left|Y_{1}-Y_{2}\right|<2.5$ correspond to the pairs in the same jet, whereas $|\Delta Y|=\left|Y_{1}-Y_{2}\right|>4$ are those for particles in opposite jets. From these figures one notices very clear manifestation of local conservation of strangeness and baryon number, apart from charge. Also that locality holds for pair of hard particles as well. Clear indication of long range correlation is seen in all pairs. In particular, clear excess of opposite charge strange pairs (fig. $\left.\underline{7}_{1}\right)$ demonstrates the leading particle effect in the two opposite jets of the $\mathrm{Z}$ decay. Using heavy flavour tagged events SLD has clearly demonstrated long range correlations. They have also observed some disagreement in data and simulation for rapidity gap correlations, particularly for pairs of different flavours. Thus the study indicates clear need to improve simulation to better describe the data in detail. These studies in turn might improve our understanding of quark asymmetry measurements, where effective hemisphere charge assignment plays important role.

It is a pleasure to thank Michiel Sanders, Eddi Wolf and David Muller for many fruitful discussions. 


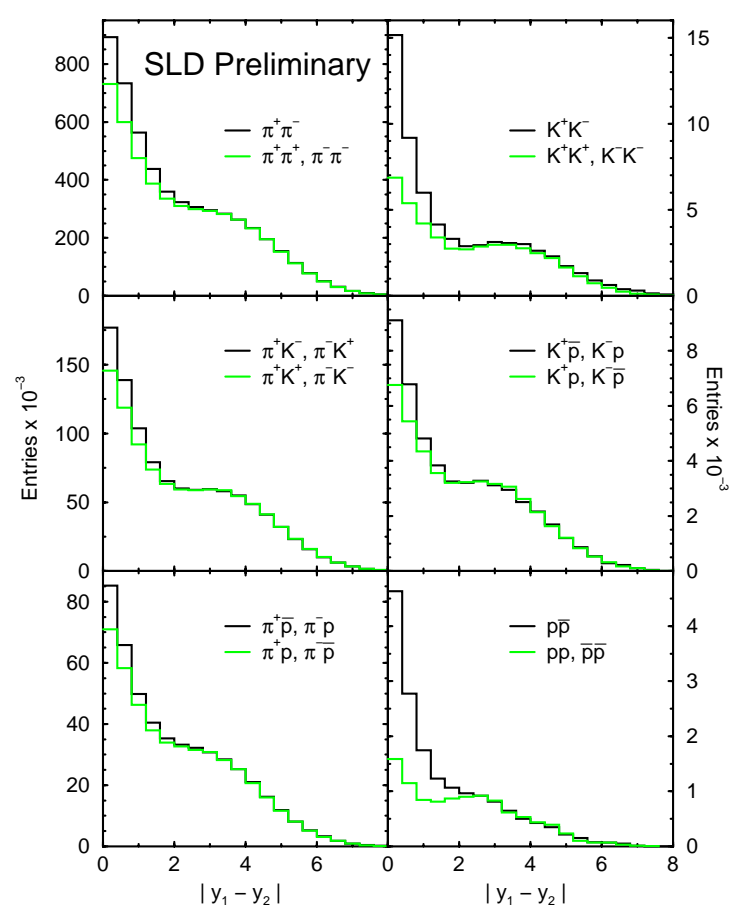

Figure 6: Rapidity gap distributions for opposite-charge and same-charge pairs of identified particles.

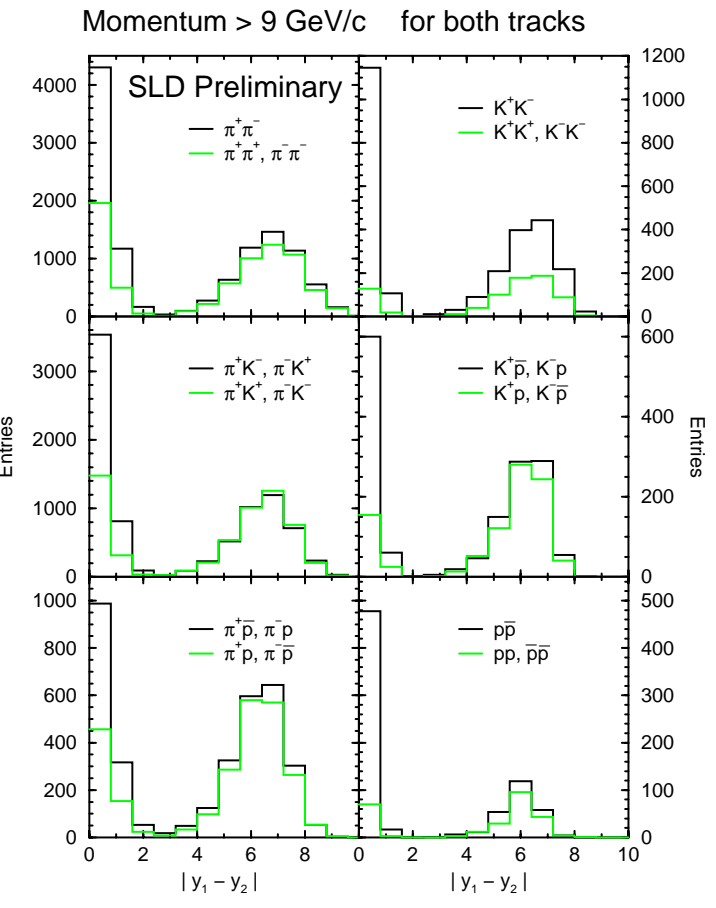

Figure 7: Rapidity gap distributions for opposite-charge and same-charge pairs for both particles having $p>9 \mathrm{GeV} / \mathrm{c}$.

\section{References}

[1] E.A. De Wolf, I.M. Dremin and W.Kittel, Phys. Rep.270 (1996) 1;

G. Alexander and H.J. Lipkin, Phys. Lett. B456 (1999) 270;

U.A. Wiedermann and U. Heinz, Phys. Rep. 319 (1999) 145;

R.M. Weiner, Phys. Rep. 327 (2000) 250;

G. Alexander Phys. Lett. B506 (2001) 45;

K. Zalewski, hep-ph/0110042.

[2] L3 Collaboration, this conference, abs \# 513, L3 note \# 2671.

[3] OPAL Collaboration, this conference, abs \# 173, OPAL note \# PN486.

[4] OPAL Collaboration, this conference, abs \# 135, OPAL note \# PN484.

[5] SLD Collaboration, this conference, abs \# 668, SLAC-PUB-8508.

[6] G. Alexander, this conference;

K. Zalewski, hep-ph/0110042.

[7] M.P. Sanders, Ph.D. Thesis, University of Nijmegen.

[8] B. Andersson and M. Ringner, Nucl. Phys. B513 (1998) 627;

B. Andersson and F. Sodergerg Eur. Phys. Jour. C16 (2000) 303.

[9] P. Carruthers and I. Sarcevic, Phys. Rev. Lett. 63 (1989) 1562.

E.A. De Wolf, Acta Phys. Pol. B21 (1990) 611.

K. Kadija and P. Seyboth, Z. Phys. C61 (1994) 465. 